\title{
OCORRÊNCIA DE MAMÍFEROS NO PARQUE ECOLÓGICO VIVAT FLORESTA - CARNÍVOROS E HERBÍVOROS
}

\author{
Mammals Occurence on Vivat Floresta Ecological Park - \\ Carnivores and Herbivores
}

\author{
Schiefelbein, $\mathrm{R}^{1}$ \\ Locatelli, I. ${ }^{2}$ \\ Russo, A. ${ }^{3}$ \\ Batalha, L M. ${ }^{4}$
}

\section{Resumo}

O levantamento de mastofauna carnívora e herbívora na área de implantação do Parque Ecológico Vivat Floresta, município de Tijucas do Sul, prevê a busca de conhecimentos básicos sobre a fauna regional. Foram utilizados os métodos indiretos de entrevista, observação de rastros nas estradas de terra entre as florestas de Pinus e em parcelas de areia. A entrevista, realizada com moradores e ex-caçadores da região, apesar de identificar 26 diferentes espécies de animais, mostrou-se de baixa confiabilidade pela falta de conhecimento específico demonstrado pelos primeiros. Nas estradas de terra foram localizados rastros de felino de pequeno porte que podem ser, segundo dados obtidos na entrevista, de Felis spp., Herpailurus yagouarundi ou Leopardus pardalis; foram encontrados ainda rastros de mustelídeos, que podem pertencer a Galictis spp. ou Lutra spp. Com relação aos herbívoros, foram observados rastros de Mazama spp. nas trilhas e, nas parcelas de areia, form identificados rastros de Dasyprocta azarae, Sylvilagus brasiliensis e Coendou prehensilis. Alguns exemplares de Sciurus spp. foram observados a olho nu. Os dados apresentados demonstram uma fauna de mamíferos carnívoros e herbívo ros variada na região, favorecendo a implantação do parque no local, apesar da existência de um reflorestamento de Pinus.

Palavras-chave: Mamíferos; Camívoros; Herbívoros; Parque Ecológico Vivat Floresta.

\section{Abstract}

The preliminary carnivore and herbivore mammals' survey in Vivat Floresta Ecological Park area, Tijucas do Sul, PR, is searching for basic knowledge on the regional fauna. Indirect methods, as interviews, animal tracks observation on land roads and sand quota were used. Although it was possible to identify 26 species, the interview, which was made with regional resident and ex-hunters, showed low confidence rate by the absence of specific knowledge presented by the regional residents. Small feline tracks were observed on land roads, and it can be from a Felis spp., Herpailurus yagouarundi or Leopardus pardalis, as it was detected in the interview data; mustelids tracks were also founded, and it can be from a Galictis spp. or Lutra spp. Tracks of Mazama spp. were observed on land roads, and the sand quota showed Dasyprocta azarae, Sylvilagus brasiliensis and Coendou prehensilis tracks. Some Sciurus spp. were observed. Those data shows a diverse carnivore and herbivore mammals fauna that can support the establishment of the Vivat Floresta Ecological Park, in spite of the existence of Pinus manmade forest.

Keywords: Mammals; Camivores; Herbivores; Vivat Floresta Ecological Park.

\footnotetext{
Aluno do Curso de Medicina Veterinária, CCAA, PUCPR - Bolsista PIBIC - CNPq.

Aluna do Curso de Zootecnia, CCAA, PUCPR

Aluna do Curso de Zootecnia, CCAA, PUCPR - Bolsista PIBIC - PUCPR

4 Professora Adjunta do Curso de Zootecnia, CCAA, PUCPR, l.batalha@pucpr.br, Rod. BR376, km 14, Bairro Costeira - CEP 83010500

- São José dos Pinhais/PR.
} 


\section{Introdução}

Entre os mamíferos existe uma variação muito grande de tamanho corpóreo, hábitos de vida e preferências de hábitat. Por isso, pesquisas e inventários de mamíferos requerem a utilização de várias metodologias específicas para diferentes grupos de espécies (VOSS; EMMONS, 1996). Dentre estes grupos, os mamíferos de médio e grande porte das florestas neotropicais, particularmente os de hábitos terrestres, têm sido pouco abordados em estudos ecológicos, especialmente no que diz respeito à composição, estrutura e dinâmica de comunidades. $\mathrm{O}$ conhecimento da biologia dessas espécies, por sua vez, tem colocado em evidência a importância desses mamíferos em uma série de processos nos ecossistemas florestais. Aparentemente, as espécies frugívoras e/ou herbívoras, como antas, veados, porcos-do-mato e roedores de grande porte, desempenham papel muito importante na manutenção da diversidade de árvores da floresta, por meio da dispersão e predação de sementes e da predação de plântulas (DIRZO; MIRANDA, 1991), ao passo que os carnívoros regulariam as populações de herbívoros e frugívoros (EMMONS, 1987; TERBORGH et al., 2001). A baixa densidade ou a extinção local de predadores de topo aparentemente leva também ao aumento de densidade de espécies de médio porte de hábitos generalistas (mesopredadores), o que pode, por sua vez, causar alterações drásticas nas comunidades de pequenos vertebrados, como aves ou pequenos mamíferos.

A fauna e a flora brasileiras vêm sendo constantemente ameaçadas pelos desmatamentos e conseqüente fragmentação das florestas, o que causa a perda de hábitat, a restrição do tamanho populacional e o isolamento de populações locais. A preocupação com os efeitos das perturbações humanas nas comunidades biológicas é ainda mais urgente com relação aos mamíferos de médio e grande porte, que necessitam de áreas comparativamente maiores e estão sujeitos à caça. A pressão de caça é, sem dúvida, uma das principias ameaças a esses animais, em áreas de floresta contínua na Amazônia, e principalmente em florestas fragmentadas como a Mata Atlântica (CULLEN et al., 2003). O grau de ameaça e a importância ecológica do grupo tornam evidente a necessidade de se incluir informações sobre os mamíferos terrestres de grande porte em inventários e diagnósticos ambientais. $\mathrm{O}$ método tradicionalmente utilizado para tais levantamentos é o censo visual em transectos lineares, muito utilizados em estudos realizados na região Amazônica, da América Central e em alguns casos na Mata Atlântica, porém, as visualizações de algumas espécies nesses estudos são muito raras (CULLEN et al., 2003). Além disso, algumas regiões florestais apresentam densidades de mamíferos mais baixas, o que torna o método de censo pouco eficaz, especialmente quando o tempo disponível para o estudo é pequeno. Outro método muito utilizado na identificação de mastofauna é por registros de pegadas em parcelas de areia que contenham iscas dispostas ao longo e fora de trilhas.

O objetivo do presente estudo foi identificar parte da mastofauna, de carnívoros e herbívoros, na área do Parque Ecológico Vivat Floresta, Município de Tijucas do Sul, por meio da observação de animais em locais predeterminados ou pela visualização de rastros em parcelas de areia e trilhas.

\section{Materiais e Métodos}

Os trabalhos de pesquisa foram conduzidos no Parque Ecológico Vivat Floresta, em uma área de 739 hectares, localizada entre os Municípios de Tijucas do Sul e Agudos do Sul, estado do Paraná. Essa área é de domínio conjunto da Associação Paranaense de Cultura (40\%), entidade mantenedora da PUCPR, e a Panagro Empreendimentos Florestais Ltda. (60\%). Situa-se entre as coordenadas UTM (22J) 7.128.500 e 7.125.500 e 673.000 e 676.500 , em uma altitude variando entre 850 e 1.200 m s.n.m., sobre o primeiro planalto paranaense e, portanto, inserido na região de domínio da Floresta Atlântica, conforme estabelece a Resolução nํ750/93 do Conselho Nacional do Meio Ambiente - CONAMA, embora predomine a fisionomia da Floresta Ombrófila Mista sensu stricto.

Para organizar o trabalho em campo, o processo foi dividido em 5 etapas básicas. Na primeira etapa foram realizadas entrevistas com a população local e ex-caçadores da região com 0 auxílio de uma apostila, contendo a descrição das características fenotípicas dos animais predominantes na área. O reconhecimento da área do parque, para identificar as trilhas existentes, corpos de água e vegetação, foi realizado na segunda etapa, assim 
como a implantação aleatória de cevas (Figura 01) para posterior avaliação e determinação dos pontos de melhor aceitação das iscas. Já na terceira etapa foram abertas duas trilhas interligadas numa distância de 100 metros por uma outra trilha já existente no local. Na quarta etapa foi efetuado registro de pegadas nas trilhas e em parcelas de areia com sua posterior identificação, sendo esta a quinta etapa. Foram distribuídas 26 parcelas ao longo das duas trilhas, demarcadas por GPS. A distância entre as parcelas variou de 10 a 65 metros, de acordo com a declividade do terreno e o tipo de vegetação. Em cada parcela foram adicionados uma banana, milho e sal mineral como isca para facilitar a atração dos animais. Três tardes por semana as pegadas em cada parcela foram fotografadas; posteriormente, a areia foi remexida para descompactar e para apagar as pegadas, permitindo novos registros; se necessário, a areia também era umedecida. A identificação das pegadas foi baseada em Becker; Dalponte (1991). Durante a quarta etapa, algumas espécies foram identificadas por visualização direta dentro do transecto. Para melhor visualização da presença dos animais na região, as porcentagens de identificação de rastros e observação de indivíduos foram separadas de acordo com a classificação taxonômica (carnívoro ou herbívoro).

\section{FIGURA 01 - Parcela de areia instalada nas trilhas com isca para atração de animais.} FIGURE 01 - Sand parcel installed in the tracks with bait for animal's attraction.

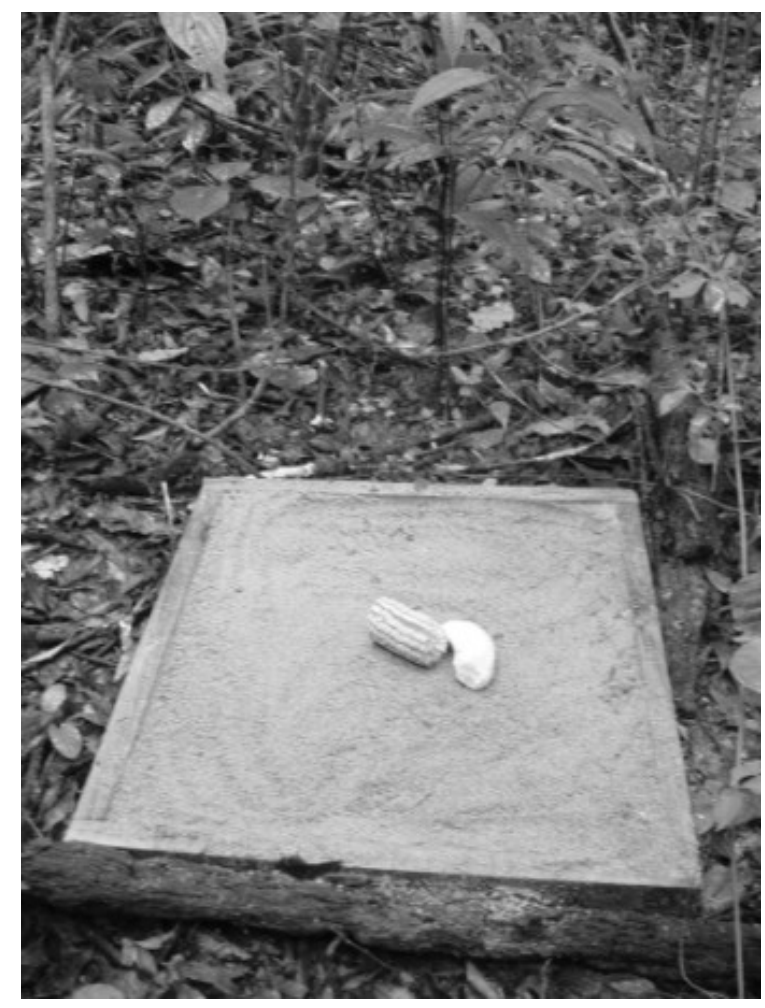

\section{Resultados}

Pela entrevista realizada com a população local e ex-caçadores, foi possível relatar a presença de 26 diferentes espécies, sendo 12 de carnívoros e 14 de herbívoros (Tabela 01). A detec- ção de rastros de carnívoros pelo método de parcelas de areia não obteve resultado significativo, mas rastros foram encontrados fora destas, como os de felídeo de pequeno porte nas trilhas (Figura 02) e de mustelídeo próximo a leitos de água (Figura 03). 
TABELA 01 - Espécies de carnívoros e herbívoros presentes na área do Parque Ecológico Vivat Floresta, Municipio de Tijucas do Sul, segundo o descrito pela população local (2004/2005). Table 01 - Carnivores and herbivores species in Vivat Floresta Eoological Park area, Tijucas do Sul, PR, as described by local population (2004/2005).

\begin{tabular}{|c|c|c|c|c|}
\hline Família & Nome comum & Nome científico & $\begin{array}{l}\text { Número de } \\
\text { relatos }\end{array}$ & Porcentagem \\
\hline Canidae & $\begin{array}{l}\text { Cachorro do } \\
\text { mato }\end{array}$ & Cerdocyion thous & 8 & 23,5 \\
\hline Canidae & Graxaim & $\begin{array}{l}\text { Dusicyon } \\
\text { gymnocercus }\end{array}$ & 2 & 5,9 \\
\hline Canidae & Raposa & Dusicyon spp. & 2 & $\begin{array}{l}5,9 \\
00\end{array}$ \\
\hline Felidae & Gato-do-mato & $\begin{array}{l}\text { Felis spp. } \\
\text { Hernailurus }\end{array}$ & 3 & 8,8 \\
\hline Felidae & Gato-mourisco & $\begin{array}{l}\text { Herpailu rus } \\
\text { vaqouarun di }\end{array}$ & 1 & 2,9 \\
\hline Felidae & Jaguatirica & Leopardus pardalis & 1 & 2,9 \\
\hline Felidae & Onça pintada & Panthera onca & 1 & 2,9 \\
\hline Felidae & Tigre $*$ & Panthera tigris & 1 & 2,9 \\
\hline Mustelidae & Furão & Galictis spp. & 2 & 5,9 \\
\hline Mustelidae & Lontra & Lutra spp. & 2 & 5,9 \\
\hline Procyonidae & Mão-pelada & Procyon crancrivorus & 2 & 5,9 \\
\hline Procyonidae & Quati & Nasua nasua & 9 & 26,6 \\
\hline \multicolumn{3}{|c|}{ TOTAL CARNIVOROS } & 34 & $100 \%$ \\
\hline Rodentia & Capivara & Hydrochoerus & 1 & 371 \\
\hline Rodentia & Paca & Agouti paca & 1 & 3,71 \\
\hline Rodentia & Serelepe & Sciurus spp. & 2 & 7,41 \\
\hline Rodentia & Nutria & Myocastor coypus & 1 & 3,71 \\
\hline Rodentia & Cutia & Dasyprocta azarae & 2 & 7,41 \\
\hline Rodentia & Preá & Cavia spp. & 1 & 3,71 \\
\hline Rodentia & Ouriço & Coen dou spp. & 1 & 3,71 \\
\hline Lagomorpha & Tapiti & Sylvilagus brasiliensis & 8 & 29,63 \\
\hline Edentata & Tamanduá Mirim & $\begin{array}{l}\text { Tamanduá } \\
\text { tetradactyla }\end{array}$ & 1 & 3,71 \\
\hline Edentata & Tamanduá & Myrmecophaga & 1 & \\
\hline Edentata & $\begin{array}{l}\text { Bandeira } \\
\text { Tatu Galinha }\end{array}$ & $\begin{array}{l}\text { tridactyla } \\
\text { Dasipus spp. }\end{array}$ & $\begin{array}{l}1 \\
1\end{array}$ & $\begin{array}{l}3,71 \\
3,71\end{array}$ \\
\hline Edentata & Tatu de Rabo & Cabassous spp. & & \\
\hline Cervidae & $\begin{array}{l}\text { Mole } \\
\text { Veado Mateiro }\end{array}$ & Mazama spp. & $\begin{array}{l}1 \\
1\end{array}$ & $\begin{array}{l}3,71 \\
3,71\end{array}$ \\
\hline Primata & Bugio & Alouatta spp. & 5 & 18,52 \\
\hline \multicolumn{3}{|c|}{ TOTAL HERBÍVOROS } & 27 & $100 \%$ \\
\hline
\end{tabular}




\section{FIGURA 02 - Pegada de felídeo de pequeno porte encontrada nas trilhas preexistentes.}

Figure 02 - Footprint of small feline founded in the preexisting tracks.

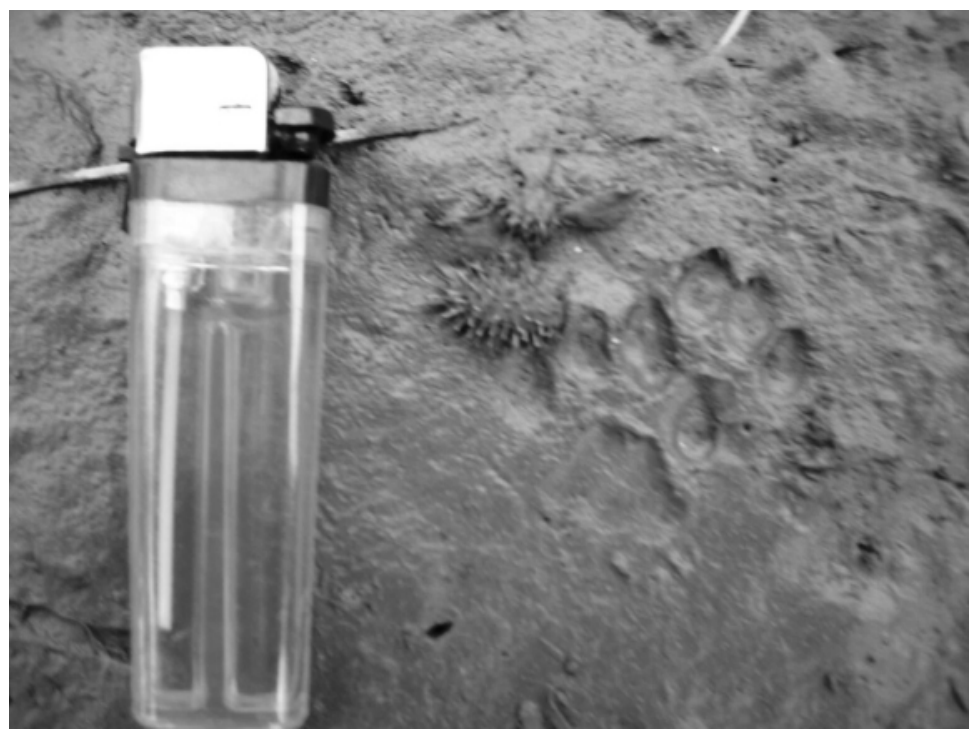

Com relação aos herbívoros, o método de parcelas de areia permitiu detectar a presença de três diferentes espécies de mamíferos herbívoros de médio porte, foram eles: Dasyprocta azarae, Coendou prehensilis e Sylvilagus brasiliensis (Figura 04). Das iscas utilizadas, a banana e o mi- lho responderam à maior parte dos registros. Nas estradas da área de Pinus foram encontradas pegadas de Mazama spp. (Figura 05), e com o método de observação a olho nu detectou-se somente o Sciurus spp.

FIGURA 03 - Pegada de mustelídeo encontrada no leito de córrego.

Figure 03 - Footprint of mustelids founded in the stream bed.

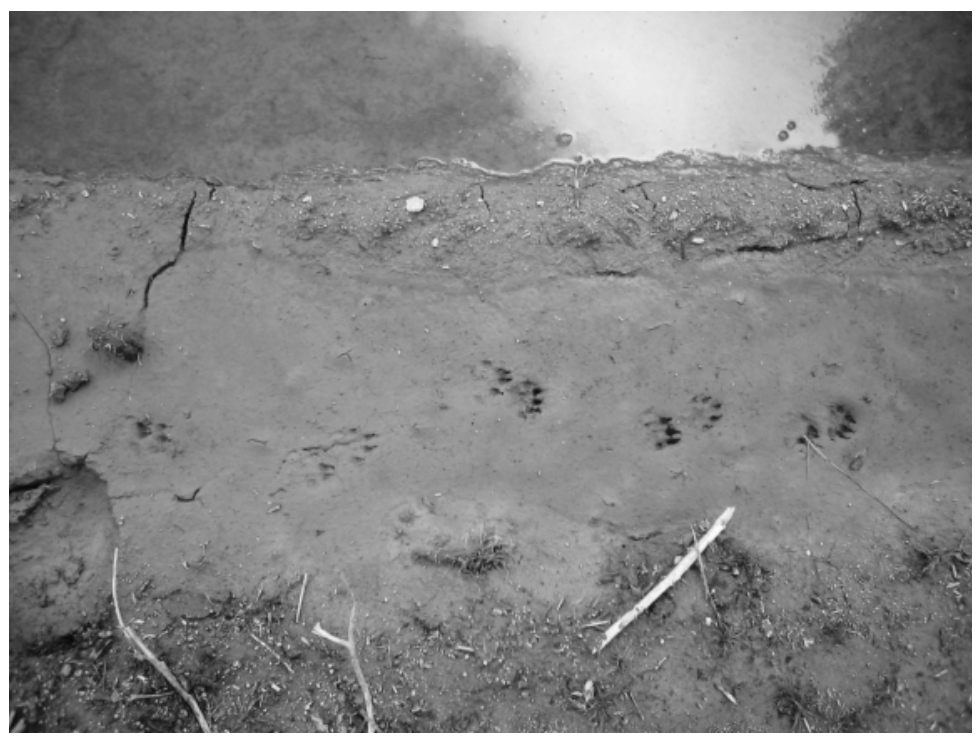


FIGURA 04 - Pegada de Sylvilagus brasiliensis deixada na parcela de areia. Figure 04 - Footprint of Sylvilagus brasiliensis in the sand parcel.

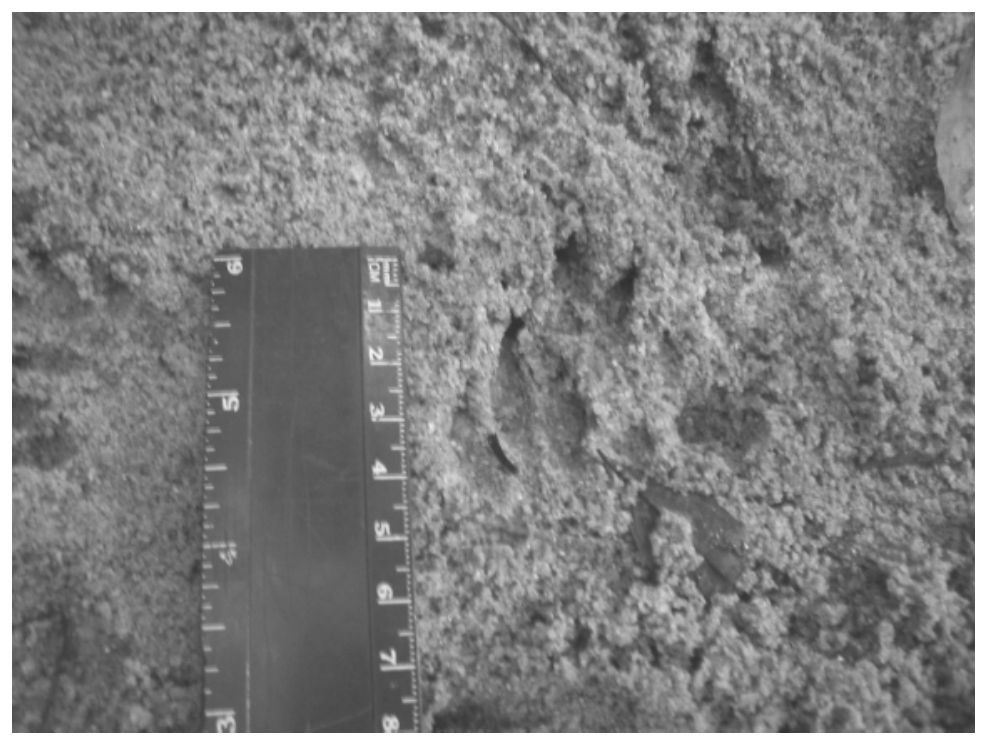

FIGURA 05 - Pegada de Mazama spp.

Figure 05 - Footprint of Mazama spp.

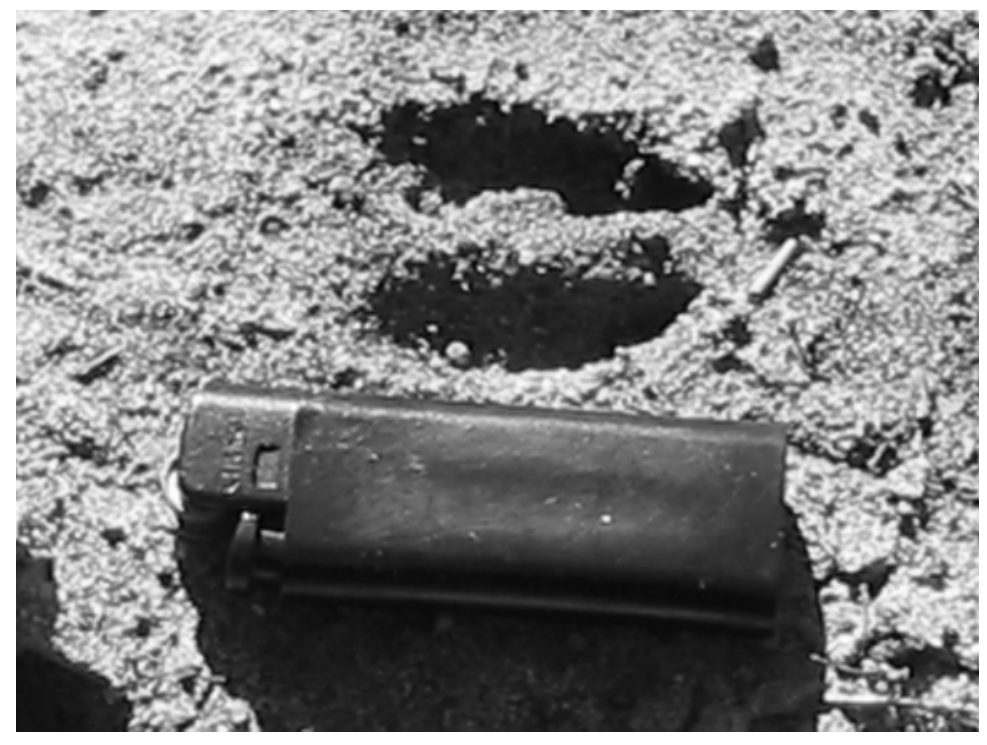




\section{Conclusões}

Para os carnívoros, a observação de rastros foi mais precisa no fornecimento de dados sobre a presença de determinadas espécies na região do que a entrevista, já que esta conta com a observação de animais por pessoas não especializadas para seu reconhecimento, vide o relato de presença de Panthera tigris, animal conhecidamente pertencente à fauna exótica. A não observação de carnívoros nas parcelas pode ter sido decorrente da falta de uso de iscas apropriadas. Para os herbívoros, os relatos obtidos pelas entrevistas mostraram-se complementares à observação de pegadas nas parcelas distribuídas ao longo das trilhas, ou seja, os animais observados estão entre os citados com maior freqüência, com exceção do Coendou spp., que foi citado poucas vezes e deixou rastros nas parcelas, e do bugio Alouatta spp. que, apesar de ser um dos mais citados, não foi observado possivelmente pelo hábito de se manter no extrato mais elevado da vegetação. $\mathrm{O}$ método de parcelas de areia mostrou-se simples e eficiente para uma breve avaliação de mastofauna herbívora, já que após um curto período de colheita de dados foram identificadas algumas espécies de maior incidência na região, inclusive algumas de hábito essencialmente noturno. $\mathrm{O}$ método mostrou-se, no entanto, falho em alguns aspectos, como: a impossibilidade de obtenção de dados a respeito do número de indivíduos por espécie, já que não permite a sua observação direta, e a falta de clareza na obtenção de pegadas em ocasiões de adversidades climáticas, como chuva. De uma forma geral foram observados rastros, nas parcelas de areia ou nas trilhas, de animais que são de grande interesse para trabalhos de conservação, visto o grau de ameaça de extinção que se encontram. Estes dados favorecem a implantação do parque no local, apesar da existência de reflorestamento de Pinus.

\section{Referências}

BECKER, M.; DALPONTE, J. C. Rastros de mamíferos silvestres brasileiros: um guia de campo. Brasília: Ed. Universidade de Brasília, 1991.

DIRZO, R.; MIRANDA, A. Contemporary neotropical defaunation and the forest structure, function and diversity - a sequel to John Terbogh. Conservation Biology, Gainesville, v. 4, p. 444-447, 1991.

EMMONS, L H. Comparative feeding ecology of felids in a Neotropical rainforest. Behavioral Ecology and Sociobiology, Berlin, v. 20, p. 271283,1987.

PARDINI, R; et al. Levantamento rápido de mamíferos terrestres de médio e grande porte. In: CULEN JR, L; RUDRAN, R.; PÁDUA, C. V. (ORG). Métodos de estudo da conservação e manejo da vida silvestre. Curitiba: Ed. da UFPR: Fundação O Boticário de Proteção à Natureza, 2003. p. 181-201.

TERBOGH, J. etal. Ecological meltdown in predatorfree forest fragments. Science, London, v. 294, p. 1923-1926, 2001.

VOSS, R. S.; EMMONS, L. H. Mammalian diversity in neotropical lowland rainforest: a preliminary assessment. Bulletin of the American Museum of Natural History, New York, v. 230, p. 1-117, 1996.
Recebido em: 21/02/2005 Aprovado em: 30/06/2005 